\title{
Sequential Everolimus and Sunitinib Treatment in Pancreatic Metastatic Well-Differentiated Neuroendocrine Tumours Resistant to Prior Treatments
}

\author{
Anna Angelousi ${ }^{a}$ Kimberly Kamp ${ }^{b}$ Maria Kaltsatou ${ }^{a} \quad$ Dermot O'Toole $^{c}$ \\ Gregory Kaltsas $^{a}$ Wouter de Herder ${ }^{b}$ \\ a Sector of Endocrinology, Department of Pathophysiology, National \& Kapodistrian University of Athens, Athens, \\ Greece; ${ }^{b}$ Sector of Endocrinology, Department of Internal Medicine, Erasmus MC, Rotterdam, The Netherlands; \\ 'St. Vincent's University and Department of Clinical Medicine, St. James Hospital and Trinity College, Dublin, Ireland
}

\section{Keywords}

Everolimus · Sunitinib · Pancreas · Molecular agents ·

Survival $\cdot$ Neuroendocrine tumour

\begin{abstract}
Objective: Alternating treatment with sunitinib and everolimus has been shown to be efficacious in renal cell carcinoma. However, no data currently exist for the role of alternate sequence administration of these agents in well-differentiated pancreatic neuroendocrine tumours (pNETs). Methods: Thirty-one patients were administered one compound and upon progression were switched to the other. All patients had grade 1 or 2 tumours and stage IV disease with similar metastatic load. The primary end point included estimation of the median overall progression-free survival (mPFS) along with each drug's mPFS as a first-line (MPFS1) and a secondline treatment (mPFS2); tolerability and serious adverse events were also evaluated. Secondary end points included overall survival (OS), 2-year mortality rate, and incidence of disease progression. Results: Overall, mPFS did not differ between the everolimus to sunitinib group (36.5 months) and the sunitinib to everolimus group (31.6 months) with a hazard ratio of 0.94 ([95\% Cl, 0.45-1.97], $p=0.7)$. Although
\end{abstract}

\section{KARGER}

(C) 2017 S. Karger AG, Basel
mPFS1 after first-line everolimus was longer (16.3 months) compared to sunitinib ( 9 months), this was not statistically significant ( $p=0.15$ ). Sequential second-line treatment showed no difference in the mPFS2 $(p=0.3)$. No difference in OS between the 2 groups was observed. Tolerability was better for everolimus compared to sunitinib. Conclusions: Treatment with sequential molecular target agents was well tolerated and associated with similar overall mPFS in both schemes of administration. Larger prospective studies are required to investigate the long-term efficacy and sequence of administration of alternate therapy with molecular targeting agents in metastatic pNETs and their effect on OS.

(c) 2017 S. Karger AG, Basel

\section{Introduction}

Digestive neuroendocrine tumours (NETs) arise from neuroendocrine cells of the pancreas (pNETs) and the gastrointestinal tract [1]. Although pNETs represent ap-

Anna Angelousi, Kimberly Kamp, Gregory Kaltsas, and Wouter de Herder contributed equally to this work. 
proximately $1.3 \%$ of all cases of pancreatic cancer, their incidence and prevalence are increasing [1]. The majority of pNETs is non-functional, and they are frequently diagnosed at a late stage in the presence of metastatic disease [1-3]. One of the largest series of patients with pNETs showed that those presenting with unresectable or metastatic disease exhibit a worse prognosis with a median overall survival (OS) of 17 months (95\% confidence interval [CI], 14-19) compared to those with localized disease that exert a median OS of 100 months (95\% CI, 68148) [2]. However, during the last years the median survival of patients with metastatic pNETs has improved substantially, reaching 41.7 months following the introduction of newer chemotherapeutic agents [4]. Although recent findings suggest that the prognosis of patients with pNETs has probably improved, more robust data on OS estimates are required.

Recently, the findings of a number of phase II and III studies have also documented the efficacy of molecular targeted therapies (MTT) in pNETs $[2,3,5]$. Everolimus inhibits the mammalian target of rapamycin (mTOR), a serine-threonine kinase that stimulates cell growth, proliferation, and angiogenesis $[3,5]$. Treatment with everolimus has obtained a $65 \%$ reduction in the estimated risk for disease progression or death compared to placebo and a significant 6.4 months increase in median progressionfree survival (mPFS) in advanced pNETs that had progressed despite previous treatment [2].

Vascular endothelial growth factor (VEGF) receptor inhibitors are also an alternative therapeutic option for patients with metastatic pNETs [6]. Sunitinib, a potent multi-targeted tyrosine kinase inhibitor with direct antitumour and anti-vascular effect, has been shown to selectively inhibit multiple receptor tyrosine kinases that are implicated in tumour growth, neoangiogenesis and metastatic progression of cancer $[7,8]$. A phase III trial that evaluated sunitinib versus placebo in patients with lowand intermediate-grade progressive pNETs found a statistically significant improvement in the PFS in the sunitinib arm (11.4 vs. 5.5 months; $p<0.001$ ) [6].

Recent position statements and guidelines propose that everolimus and sunitinib are both approved in progressive grade 1 and 2 pNETs, irrespective of Ki-67 value and tumour burden, and are considered as different treatment options in pNETs [9]. Thus, both agents may be used as first- or second-line options with respect to chemotherapy or subsequent to therapy with long-acting somatostatin analogues (SSAs) [9-11].

These 2 agents are standard therapies for other types of cancer such as metastatic renal carcinoma $[12,13]$.

Everolimus and Sunitinib Sequential

Treatment in pNETs
Current treatment guidelines for patients with metastatic renal cell cancer recommend a first-line challenge with a VEGF inhibitor, including sunitinib, followed by everolimus at progression $[12,13]$. Recent trials have shown the non-inferiority of everolimus compared to sunitinib as first-line treatment and support the standard treatment paradigm of first-line sunitinib followed by everolimus at progression [14].

In the present study, we aim to investigate whether MTT with everolimus and sunitinib administrated sequentially in patients with advanced well-differentiated pNETs, that developed disease progression following the administration of one of these agents, could be tolerated and affect disease progression and survival.

\section{Patients and Methods}

\section{Patients}

We studied 31 patients with advanced well-differentiated pNETs and stage IV/IIIb disease at initial diagnosis and evaluated their response to sequential treatment with MTT according to Response Evaluation Criteria in Solid Tumours (RECIST) criteria. Before entering the study, all patients had disease progression documented by RECIST besides different prior therapeutic modalities (surgery, peptide receptor radiotherapy treatment [PRRT], chemotherapy and SSA). In particular, 18 patients had already received PRRT (median 6 [range 3-9] cycles), 12 patients had already received chemotherapy ( 1 patient had received 4 cycles of combination therapy with capecitabine/temozolomide, 6 patients streptozotocin and 5-fluorouracil, 2 patients cisplatin-etoposide, and 3 patients temozolomide alone), and 22 patients were treated with SSAs concomitantly with other treatments.

All patients had stage IV disease at the time of initiation of MTT, similar tumour load, in respect of the different organs involved (liver, chest, bone, lymph nodes), the number and the size of metastases, and similar grading as shown by the Ki-67\% labelling index value. All patients of each group entered the second-line treatment with MTT when the disease had progressed according to findings of imaging modalities including computed tomography and magnetic resonance imaging or when there was no tolerability of MTT because of serious adverse events (AEs). Radiological imaging to document disease progression was performed every 3-6 months.

Patients had no contraindications for the initiation of MTT or other serious comorbidities except well-controlled hypertension in 6 patients and diabetes mellitus type 2 in 8 patients ( 3 out of 31 were receiving concomitantly at least 1 anti-hypertensive drug and/or 1 anti-diabetic agent). Before treatment initiation with an MTT, all patients had pre-defined haematology and biochemistry analysis (baseline values) which included a complete blood count control, urea and electrolytes, renal and hepatic function, bone metabolism, and thyroid function.

Study Design and Treatment

In this retrospective study, data were obtained and analyzed from 2 institutional collected databases from 2 centres. The study

Neuroendocrinology 2017;105:394-402 DOI: 10.1159/000456035 
included consecutive patients with metastatic pNETs (stage IV) resistant to prior therapeutic modalities who received an MTT, either everolimus $(n=20)$ as first-line treatment followed by sunitinib as second challenge (everolimus to sunitinib group) or sunitinib $(n=11)$ as a first-line treatment followed by everolimus (sunitinib to everolimus group) as second challenge. The allocation of the sequence of treatment with either MTT was decided by the treating physicians based on local availability and personal preferences.

Patients received each drug until documented disease progression according to RECIST criteria or development of unacceptable toxicity. The everolimus full dosage was $10 \mathrm{mg}$ daily, and the sunitinib $37.5 \mathrm{mg}$ daily. However, dose modifications were permitted in the presence of AEs, so that everolimus could be decreased to $5 \mathrm{mg}$ and sunitinib to $25 \mathrm{mg}$ daily, respectively.

The study protocol was approved by the ethics committee of both participating centres, according to the 3rd edition of the Guidelines on the Practice of Ethical Committees in Medical Research. All patients gave informed consent according to the Declaration of Helsinki and Good Clinical Practice guidelines.

\section{Primary and Secondary End Points}

The primary end point of the study was to assess overall mPFS after the introduction of both agents in each treatment group, as well as the mPFS after first- and second-line treatment with either MTT agent used in a sequential manner. In particular, mPFS after the first-line treatment (mPFS1) was defined as the time from the first initiation of an MTT (with either everolimus or sunitinib) to treatment discontinuation either due to disease progression or development of serious AEs. MPFS for the second-line treatment (mPFS2) was defined as the time after the switch from one MTT agent to the other, until treatment discontinuation either due to disease progression or development of serious AEs or death.

Tolerability and AEs were also included as primary end points. AEs were classified as grade 1 (mild and transient effect), grade 2 (moderate), grade 3 (severe necessitating medical intervention), and grade 4 (potentially life threatening necessitating hospitalization). Dose modifications were introduced for grade 1,2 , and 3 AEs. Discontinuation of the treatment was decided when the patients had progressed according to RECIST or when patients developed unacceptable toxicity (grade 3 or 4 ).

Secondary end points included estimation of OS in months defined as the time from the initiation of the MTT to death as well as the 2-year survival rate. Incidence of the disease progression was also analyzed. Hazard ratios (HR) were calculated for both the PFS and OS.

\section{Statistical Analysis}

Statistical analysis was performed using Stata 10 (StataCorp, College Station, TX, USA). The survival analysis was performed using the Kaplan-Meier method, and the results were compared using the log-rank test. Cox proportional hazard models (HR) with $95 \%$ CI were applied adapted for confounding factors such as age and gender. Results were considered significant at $p<0.05$. The distribution of continuous variables is reported as median values or mean \pm standard deviation (SD). Recurrent events analysis was performed using the Andersen-Gill Cox regression model [15].

\section{Results}

\section{Patient Population}

Thirty-one patients (8 females, median age 52.5 years) were recruited from 2 European Centres (Department of Pathophysiology, Sector of Endocrinology, Laiko Hospital, University of Athens, Greece, and Department of Internal Medicine, Sector of Endocrinology, Erasmus MC, Rotterdam, Netherlands). The characteristics of the studied population are presented in Table 1. Patients had no differences in respect of their baseline characteristics such as age, sex, grade, and disease stage. Ten patients (32\%) had a functional pNET, $18 \%$ in the sunitinib to everolimus group, and $40 \%$ in the everolimus to sunitinib group albeit with no statistical significant differences $(p=0.2)$. Twenty-three patients had stage IV and 4 patients had stage IIIb disease at initial diagnosis; however, at the initiation of MTT, all patients had stage IV disease. Nineteen (61\%) patients had grade 2 and 8 (26\%) grade 1 tumours (in the remaining 4 , the grade was unknown). Twenty patients received first-line everolimus followed by sunitinib, and 11 were assigned to first-line sunitinib followed by everolimus. The most frequent previous therapeutic modalities included SSAs (in 71\% of the cases), PRRT (in $58 \%$ of the cases), surgical ablation (in $58 \%$ of the cases), and chemotherapy (in 39\% of the cases). PRRT was a more frequent treatment modality in the everolimus to sunitinib group versus sunitinib to everolimus group ( 70 vs. $36 \%, p=0.07$ ), whereas liver-directed therapy in the form of radiofrequency ablation was more frequent in the sunitinib to everolimus group (27 vs. $5 \%, p=0.08)$, albeit without both reaching statistical significance (Table 1).

Tumour load and distribution were similar in all patients. The median $\mathrm{Ki}-67$ value was $5 \%$ in both groups (mean values $=6.0 \pm 3.5$ for the everolimus to sunitinib group vs. $5.0 \pm 3.9$ for the sunitinib to everolimus group, $p=0.33$ ). Seven patients had a Ki-67 $>10 \%$ and 20 had a Ki-67 $<10 \%$, whereas in 4 patients the Ki- 67 was not available. All 20 patients from the group that received first-line everolimus had liver metastases; 13 of these patients (65\%) had multiple ( $>5)$ metastases, 4 also had bone metastases, and 2 had pulmonary metastases. All 11 patients from the first-line sunitinib group had liver metastases; in 7 of them $(63 \%)$, the metastases were multiple $(>5), 1$ also had bone metastases, 1 peritoneal metastases, and 1 had pulmonary metastases.

The time interval between the first-line treatment and the second challenge during which the vast majority of
Angelousi/Kamp/Kaltsatou/O’Toole/ Kaltsas/de Herder 
Table 1. Baseline patient characteristics and comparison of the patients in the 2 studied groups (sunitinib to everolimus vs. everolimus to sunitinib)

\begin{tabular}{|c|c|c|c|c|}
\hline Characteristics & Whole group & $\begin{array}{l}\text { Sunitinib to } \\
\text { everolimus group }\end{array}$ & $\begin{array}{l}\text { Everolimus to } \\
\text { sunitinib group }\end{array}$ & $p$ value \\
\hline Patients, $n$ & 31 & 11 & 20 & \\
\hline Median age, years & 52.5 & 51.46 & 57.9 & 0.3 \\
\hline Males, \% & 23 & $9(81 \%)$ & $14(80 \%)$ & 0.5 \\
\hline Stage & all stage IV & all stage IV & all stage IV & \\
\hline \multicolumn{5}{|l|}{ Grade } \\
\hline 1 & $8(26 \%)$ & $4(36 \%)$ & $4(25 \%)$ & 0.8 \\
\hline 2 & $19(61 \%)$ & $6(55 \%)$ & $13(65 \%)$ & 0.8 \\
\hline Unknown & $4(13 \%)$ & $1(1 \%)$ & $3(15 \%)$ & \\
\hline Familial syndrome & $4(13 \%)$ & 1 (MEN-1) & 3 (all MEN-1) & \\
\hline Functional syndrome & $10(32 \%)$ & $2(18 \%)$ & $8(40 \%)$ & 0.2 \\
\hline \multicolumn{5}{|l|}{ Other comorbidities } \\
\hline HTA & $6(19 \%)$ & - & - & \\
\hline DM2 & $8(26 \%)$ & - & - & \\
\hline \multicolumn{5}{|l|}{ Previous treatments } \\
\hline Surgery & $18(58 \%)$ & $7(63 \%)$ & $11(55 \%)$ & 0.6 \\
\hline Chemotherapy & $12(39 \%)$ & $4(36 \%)$ & $8(40 \%)$ & 0.8 \\
\hline PRRT & $18(58 \%)$ & $4(36 \%)$ & $14(70 \%)$ & 0.07 \\
\hline SSA & $22(71 \%)$ & $8(72 \%)$ & $13(65 \%)$ & 0.65 \\
\hline TACE & $3(10 \%)$ & $1(9 \%)$ & $2(10 \%)$ & 0.4 \\
\hline Radiofrequency & $4(13 \%)$ & $3(27 \%)$ & $1(5 \%)$ & 0.08 \\
\hline Ki-67, \% & $6 \pm 0.5(5)$ & $5.0 \pm 3.9(5)$ & $6.0 \pm 3.5(5)$ & 0.33 \\
\hline Follow-up, months & & $11.7 \pm 11.8(7.4)$ & $14.3 \pm 12.9(10.4)$ & 0.6 \\
\hline PFS for pNETs ${ }^{\mathrm{a}}$ & $12.5(7.7-27.7)$ & $9(6.0-9.4)$ & $16.3(7.8-27.7)$ & 0.15 \\
\hline PFS for $\mathrm{pNETs}^{\mathrm{b}}$ & $12.4(3.4-22.6)$ & $15.5(4.3-25)$ & $10.4(2.4-13.3)$ & 0.3 \\
\hline \multicolumn{5}{|l|}{ Disease progression } \\
\hline After first-line treatment & $25(81 \%)$ & $7(63 \%)$ & $18(90 \%)$ & 0.3 \\
\hline After second-line treatment & $16(52 \%)$ & $7(55 \%)$ & $9(60 \%)$ & 0.4 \\
\hline Mortality & $11(35 \%)$ & $3(27 \%)$ & $8(40 \%)$ & 0.5 \\
\hline
\end{tabular}

HTA, hypertension; DM2, diabetes mellitus type 2; PPRT, peptide receptor radiotherapy; SSA, somatostatin analogues; TACE, transarterial chemo-embolization; PFS, progression-free survival; pNET, pancreatic

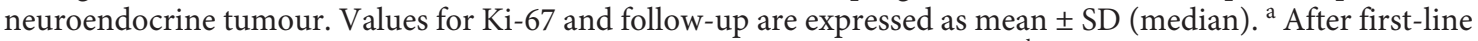
treatment with everolimus or sunitinib (before the switch); median (25-75\%). ${ }^{\mathrm{b}}$ After second-line treatment with everolimus or sunitinib (after the switch); median (25-75\%).

patients received no other treatment except SSAs was similar in the 2 groups: $3.92 \pm 5.0$ months for the everolimus to sunitinib group versus $4.08 \pm 5.9$ months for the sunitinib to everolimus group $(p=0.78)$.

\section{Primary End Points}

Progression-Free Survival

No significant differences were found for overall mPFS in both treatment groups. In particular, the overall mPFS for the everolimus to sunitinib group was 31.6 months and for the sunitinib to everolimus 36.5 months with an HR of 0.94 (95\% CI, 0.45-1.97), suggesting that the order of the sequencing of the treatment did not exert a significant effect on mPFS. The mPFS1 after first-line everolimus was longer (16.3 months, $n=20)$ compared to sunitinib (9 months, $n=11$ ), although not statistically significant ( $p=0.15)$ with an HR of 1.98 (95\% CI, 0.77-5.14) for the first-line sunitinib vs. the everolimus group (Fig. 1). The mPFS2 after the sequential second-line treatment also showed no difference $(\mathrm{p}=0.3)$ between the 2 agents (15.5 months for everolimus, $n=11$, vs. 10.3 months for sunitinib, $n=20)$ with an HR of 1.66 (95\% CI, $0.63-4.36$ ) for the second-line sunitinib vs. the everolimus group (Fig. 2). 


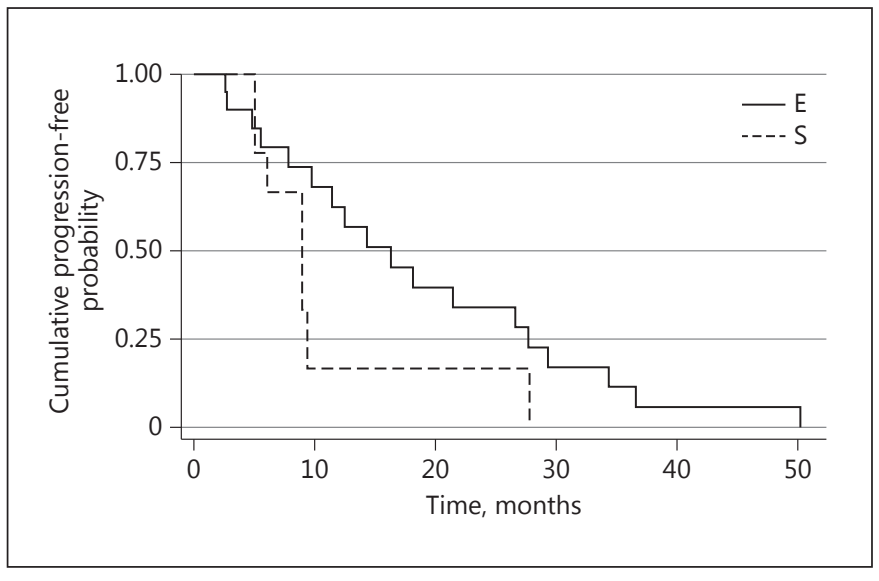

Fig. 1. Kaplan-Meier plots for progression-free survival (PFS) after first-line treatment with everolimus (E) and first-line treatment with sunitinib (S). The median PFS1 for the first-line treatment with everolimus was 16.3 months, and with sunitinib 9 months $(p=0.15$, HR $[95 \% \mathrm{CI}]=1.98[0.77-5.14])$.

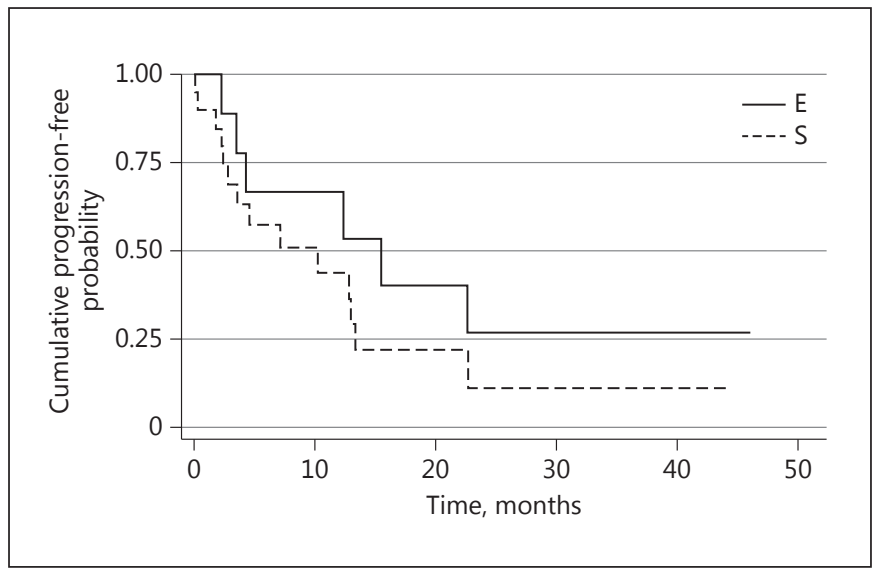

Fig. 2. Kaplan-Meier plots for progression-free survival (PFS) after second-line treatment with everolimus (E) and sunitinib (S). The median PFS2 after the sequential second-line treatment showed no difference ( $p=0.3$ ) between the 2 agents ( 15.5 months for everolimus vs. 10.3 months for sunitinib, HR $[95 \% \mathrm{CI}]=1.66[0.63-4.36])$.

Table 2. Number of patients who developed AEs following MTT with everolimus and sunitinib during the first-line and the second-line treatment

\begin{tabular}{|c|c|c|c|c|c|c|c|c|}
\hline \multirow[t]{2}{*}{ AEs } & \multicolumn{2}{|c|}{$\begin{array}{l}\text { 1st line everolimus } \\
(n=20)\end{array}$} & \multicolumn{2}{|c|}{$\begin{array}{l}\text { 1st line sunitinib } \\
(n=11)\end{array}$} & \multicolumn{2}{|c|}{$\begin{array}{l}\text { 2nd line everolimus } \\
(n=11)\end{array}$} & \multicolumn{2}{|c|}{$\begin{array}{l}\text { 2nd line sunitinib } \\
(n=20)\end{array}$} \\
\hline & grade $1 / 2$ & grade 3 & grade $1 / 2$ & grade 3 & grade $1 / 2$ & grade 3 & grade $1 / 2$ & grade 3 \\
\hline Haematological $^{\mathrm{a}}$ & $4(20)$ & $1(5)$ & $2(18)$ & $2(18)$ & $2(18)$ & $1(9)$ & $4(20)$ & $2(10)$ \\
\hline \multicolumn{9}{|l|}{ Gastrointestinal } \\
\hline intolerance $\mathrm{e}^{\mathrm{b}}$ & $3(15)$ & - & $4(36)$ & $2(18)$ & $3(36)$ & $1(9)$ & - & $2(10)$ \\
\hline Fatigue & $2(10)$ & - & $3(36)$ & $2(18)$ & $2(18)$ & - & $2(10)$ & $2(10)$ \\
\hline Liver dysfunction & $2(10)$ & - & - & - & $1(9)$ & - & - & $1(5)$ \\
\hline Abdominal pain & - & - & $2(18)$ & $1(9)$ & - & - & $1(5)$ & $3(15)$ \\
\hline Dermatitis & $5(25)$ & - & $1(9)$ & - & $2(18)$ & - & $1(5)$ & - \\
\hline Hyperglycaemia & $4(20)$ & $2(10)$ & - & - & $2(18)$ & - & - & - \\
\hline Pneumonitis & $4(20)$ & $1(5)$ & - & - & $1(9)$ & $1(9)$ & - & - \\
\hline Kidney failure & $2(10)$ & - & $1(9)$ & - & $1(9)$ & - & $1(5)$ & - \\
\hline Hypothyroidism & $1(5)$ & - & - & - & - & - & $4(20)$ & - \\
\hline Stomatitis & $2(10)$ & - & $3(36)$ & - & $1(9)$ & - & $1(5)$ & $1(5)$ \\
\hline
\end{tabular}

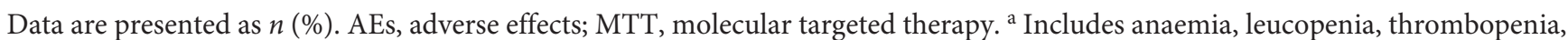
${ }^{\mathrm{b}}$ Includes nausea, vomiting, diarrhoea, change of taste.

\section{Adverse Events and Tolerance}

Fifteen out of $20(75 \%)$ patients developed AEs (all grades) during the first-line treatment with everolimus compared to 7 patients out of 11 (64\%) during the firstline treatment with sunitinib. Five out of 11 (45\%) developed AEs (all grades) during the second-line treatment with everolimus compared to 11 out of $20(55 \%)$ during the second-line treatment with sunitinib. Four out of 20
(20\%) patients who received first-line treatment with everolimus had grade $3 \mathrm{AEs}$, and 2 of them (10\%) had to discontinue treatment with everolimus and switch to the second challenge treatment with sunitinib because of intolerant AEs (Table 2). Five out of $11(45 \%)$ patients who received first-line treatment with sunitinib had grade 3 AEs, and 4 of them (36\%) had to switch to the second challenge treatment with everolimus because of intoler- 


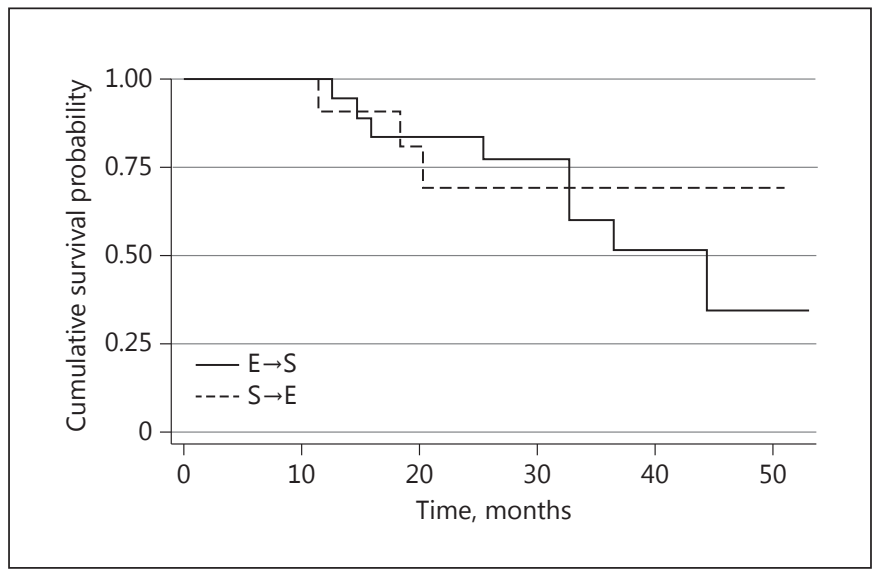

Fig. 3. Kaplan-Meier plots for overall survival (OS) for the everolimus to sunitinib group $(\mathrm{E} \rightarrow \mathrm{S})$ compared to sunitinib to everolimus group $(S \rightarrow E)$. No statistical significant differences were found in the median OS time between both groups ( $p=0.875$, HR [95\% $\mathrm{CI}]=0.90[0.23-3.45])$.

ant AEs. During the second-line treatment, 3 out of 11 (27.7\%) patients who received everolimus as second-line treatment had grade 3 AEs, and 1 of them (9\%) discontinued treatment. Seven out of the $20(35 \%)$ patients who received sunitinib as second-line treatment had grade 3 AEs, and $3(15 \%)$ of them discontinued treatment (Table 2).

None of the patients developed grade 4 AEs. In all the other cases, AEs were well tolerated especially after dose adaptation. Serious AEs after first-line treatment with either single agent were less compared to those after the sequential treatment with the 2 agents (serious AEs presented in $10 \%$ of patients who received everolimus as first-line treatment vs. $25 \%$ of patients who received the sequential treatment of everolimus followed by sunitinib, $p=0.4$, and in $36 \%$ of patients who received sunitinib as first-line treatment vs. $45 \%$ of patients who received the sequential treatment of sunitinib followed by everolimus, $p=1.0)$.

Anaemia, hyperglycaemia and pneumonitis as well as cutaneous manifestations were the most frequent AEs in the everolimus to sunitinib group, whereas fatigue and abdominal pain in the sunitinib to everolimus group.

\section{Secondary End Points}

No statistically significant differences were found in the median OS time in both groups ( $p=0.875)$ (Fig. 3). The HR for the sunitinib to everolimus group versus the everolimus to sunitinib group was 0.90 (95\% CI, 0.23 $3.45)$. The 2 -year survival rate in the everolimus to suni-

Everolimus and Sunitinib Sequential Treatment in pNETs tinib group was 0.83 (95\% CI, 0.57-0.94) compared to 0.69 (95\% CI, 0.31-0.89) in the sunitinib to everolimus group, but without statistical significance.

Disease progression after the first-line treatment was observed in 18 out of 20 patients with pNETs (90\%) after a median 13.6 months' exposure to everolimus and in 7 out of 11 patients (63\%) after a median 7.4 months' exposure to sunitinib. Disease progression after the secondline treatment was observed in 9 out of 20 patients (45\%) after a median 7.2 months' exposure to sunitinib and in 7 out of 11 patients (63\%) after a median 7 months' exposure to everolimus. Second-line treatment was discontinued because of death in 8 patients (40\%) from the everolimus to sunitinib group and in 3 patients (27\%) from the sunitinib to everolimus group. The overall mortality rate for both groups was 35\% (11 patients out of 31). All deaths were considered disease-related and were observed only during the second-line treatment period with either agent.

\section{Discussion}

This is the first study in metastatic pNETs and progressive disease despite previous treatments investigating the tolerability and the role of sequential treatment using the currently approved MTT agents, everolimus and sunitinib, in respect of PFS and OS. The comparison of first-line treatment with either everolimus or sunitinib did not show any statistical difference in PFS in favour of any of the 2 agents used. In addition, sequential treatment was not associated with any difference in OS between the 2 therapeutic schemes. Serious AEs necessitating treatment discontinuation were observed in $25 \%$ of patients in the everolimus to sunitinib group and in $45 \%$ of patients in the sunitinib to everolimus group. Patients seemed to tolerate better everolimus compared to sunitinib albeit without statistical significance.

The PFS obtained when either agent was used as second-line treatment compares favourably to that obtained in the placebo group of patients included in the RADIANT-2 and the study evaluating the efficacy of sunitinib in progressive pNETs $[6,11]$. Although indirect, these findings suggest that sequential administration of MTT agents results in clinically meaningful responses with prolongation of PFS following disease progression to the initial challenge with MTT. Although there was no statistical significance in overall mPFS between the 2 schemes, everolimus as first-line treatment was associated with a better mPFS1 compared to sunitinib (16.3 vs. 9.0 months,

Neuroendocrinology 2017;105:394-402 DOI: 10.1159/000456035 
respectively), whereas sunitinib as second-line treatment was associated with a better mPFS2 compared to everolimus (15.5 vs. 10.4 months, respectively). A possible continuous anti-proliferative effect of previously applied PRRT that was mostly utilized in the everolimus to sunitinib group could have accounted for this effect; however, patients in the sunitinib to everolimus group had more liver-directed cytoreductive interventions. Although robust conclusions regarding the ideal sequence of treatment cannot be derived from this study, it is probable that previous treatments could affect first-line treatment and the efficacy of the sequential treatment. It is expected that the on-going characterization of the molecular profiling of pNETs will make possible the selection of the sequence of the most appropriate agent [16].

pNETs are in their great majority slowly growing tumours and generally more indolent than adenocarcinomas; however, once these tumours progress beyond surgical resectability, they are essentially incurable [17]. Systemic treatment options have substantially been expanded in recent years with long-acting SSAs regarded as the first therapeutic option for well-differentiated pNETs with a Ki-67 value $<10 \%$ [18]. However, the majority of patients will eventually develop progressive disease necessitating further systemic therapy. Following disease progression, different therapeutic options are available including cytotoxic chemotherapy, PRRT and the administration of MTT agents [19, 20]. Currently, there are no comparative studies evaluating the ideal sequence of these therapies upon progression, although several algorithms have been proposed by relevant scientific societies taking into consideration parameters such as the $\mathrm{Ki}-67$ value, tumour growth rate, and disease burden $[10,19]$.

Although MTT agents have been used following disease progression to SSAs, the issue of sequential treatment with currently available MTT agents in pNETs has not yet been formally addressed as in other malignancies [10]. Alternate sequence of sunitinib and everolimus has been well studied in advanced renal cell carcinoma where sunitinib and everolimus are both approved as first-line and second-line therapies, respectively $[12,13]$. A prospective clinical trial in patients with advanced renal cancer (RECORD-3) comparing sunitinib until progression followed by everolimus versus the reverse sequence demonstrated the non-inferiority of everolimus compared to sunitinib in terms of PFS and OS, with important however crossover of patients (43-45\%) from one group to the other [14]. Sunitinib exhibited a slightly longer PFS and OS without reaching statistical significance, though
[14]. In the RECORD-1 trial, that compared everolimus with placebo after sunitinib or sorafenib failure in advanced renal cell carcinoma, the mPFS was 4.9 vs. 1.9 months compared to the placebo group [21]. These data suggest that a programmed sequential strategy administering sunitinib and everolimus without waiting for disease progression may be considered as a potential option in patients with advanced renal cell carcinoma. In the present study, sequential therapy with both MTT schemes showed increased PFS compared to monotherapy with each agent alone, without the sequence of administration being important.

Although there are no clinical data evaluating the effects of a second-line challenge treatment with an MTT agent in pNETs, in vitro studies offer a better understanding of the mechanism underlying the role of the sequence of treatment of MTT agents in cancerous tissues. The sequential combinations of everolimus and sunitinib in a renal cancer mouse model induced anti-angiogenic effects, leading to tumour necrosis [22]. In particular, the addition of everolimus after 5 weeks' exposure of renal cancer cells to sunitinib slowed tumour growth further [22]. Alternate sequence of sunitinib and everolimus mitigated the development of mesenchymal phenotype compared with sunitinib as single agent [23]. In particular, mice treated with sunitinib monotherapy presented controlled tumours characterized by lower vessel size compared to progressive tumours, while everolimus was more efficient on mature vessels than sunitinib that mainly affected unstable neoangiogenesis [23].

Besides sequential treatment, multiple combination therapies have been explored in phase I/II trials either as a de novo combination of 2 targeted drugs, or of targeted drugs with SSAs, but also with chemotherapy, PRRT, or even locoregional therapies of the liver. In one of these trials, everolimus was explored in combination with the novel SSA pasireotide, an upstream activator of the mTOR pathway without any benefit in the PFS [24]. Another trial investigated the combination of everolimus and the angiogenesis inhibitor bevacizumab that binds circulating VEGF compared to everolimus alone [25]. Although PFS was statistically significantly different between the 2 arms, the clear benefit was only modest ( 2.7 months) in the combination treatment of everolimus with bevacizumab, at the expense of more side effects. Due to more side effects, treatment was discontinued in $38 \%$ of the patients receiving the combination treatment compared to $16 \%$ with everolimus alone [25]. Another promising combination therapy is that of metformin in association with everolimus. Metformin activates ade-
400

Neuroendocrinology 2017;105:394-402 DOI: $10.1159 / 000456035$
Angelousi/Kamp/Kaltsatou/O’Toole/ Kaltsas/de Herder 
nosine monophosphate-activated protein kinase, and might enhance the anti-proliferative efficacy of everolimus. Both drugs are currently investigated in pNETs along with the administration of octreotide [26].

While the choice of targeted therapies in other malignancies is frequently driven by the findings of the precise molecular alterations present in the tumour, no such study has been done in NETs. This is particularly important given that the survival of patients with advanced NETs appears to be different based on the driver mutation(s) present in the tumour. An ongoing study includes the analysis of the involved cell signalling pathways for the treatment of patients with advanced metastatic gastrointestinal NETs and pNETs with currently approved MTT agents according to the mutations present (sunitinib for MEN1/PDGFR/KIT/FLT3 mutations or everolimus for NF1/PTEN/PI3K/AKT/mTOR/VHL/TP53 mutations) [16].

In this study, both combinations with the 2 MTT agents were relatively well tolerated by the majority of the patients. When comparing the tolerability of each agent when administered alone as first-line treatment with other studies, dermatitis of any grade was the most frequent $\mathrm{AE}(25 \%)$ in our patients treated with first-line everolimus, and hyperglycaemia was the most serious one (10\%) (grade 3). However, in the RADIANT-2 [11] and RADIANT-4 [27] trials, stomatitis (7 and 9\%, respectively) was the most frequent and the most serious $\mathrm{AE}$ followed by diarrhoea (6 and 7\%). In addition, diarrhoea, fatigue, and stomatitis were the most frequent AEs (36\%) in our patients treated with first-line sunitinib, and neutropenia, diarrhoea, and fatigue ( $18 \%$ for each symptom) the most serious ones (grade 3/4). Similarly, in the SUN III [6] study, diarrhoea was also the most frequent AE of any grade (59\%) and neutropenia (12\%) the most frequent serious AE (grade $3 / 4$ ).

The major inherent limitation of the present study lies, due to its design, in the retrospective nature of the analysis. This limitation, a common feature in most observational studies, may explain the allocation of a different number of patients in each group and the different duration of treatment. Unfortunately, the small sample size did not provide sufficient statistical power for any of our findings. Additionally, the lack of a separate group of patients receiving monotherapy with an MTT agent did not permit a direct comparison between the single agent treatment and the combination of the 2 agents regarding PFS, OS, and mortality. However, this is the first clinical study comparing sequential challenge treatment in this rare category of neoplasms and showing that this treat-

Everolimus and Sunitinib Sequential

Treatment in pNETs ment is well tolerated and can be used even in previously heavily treated patients. The rationale of performing such studies has also been highlighted in a recent comprehensive review of the effect of available MTT on PFS and OS in patients with pNETs [28].

In conclusion, overall mPFS and OS did not seem to differ significantly among patients who received sequential treatment with both everolimus and sunitinib irrespective of the order of administration. The sequential treatment was well tolerated in both schemes of administration, but the small sample size and the retrospective nature of our study did not allow a robust conclusion to be made. However, these data provide evidence that programmed alternating sequential strategies studied in a prospective manner could demonstrate the therapeutic efficacy of this approach in patients with advanced pNETs.

\section{Funding Sources}

This research did not receive any specific grant from any funding agency in the public, commercial, or not-for-profit sector.

\section{Disclosure Statement}

There is no conflict of interest that could be perceived as prejudicing the impartiality of the research reported.

References

1 Huguet I, Grossman AB, O’Toole D: Changes in the epidemiology of neuroendocrine tumours. Neuroendocrinology 2017;104:105111.

2 Halfdanarson TR, Rabe KG, Rubin J, Petersen GM: Pancreatic neuroendocrine tumours (pNETs): incidence, prognosis and recent trend toward improved survival. Ann Oncol 2008; 19:1727-1733.

3 Yao JC, Shah MH, Ito T, Bohas CL, Wolin EM, Van Cutsem E, Hobday TJ, Okusaka T, Capdevila J, de Vries EG, Tomassetti P, Pavel ME, Hoosen S, Haas T, Lincy J, Lebwohl D, Öberg K; RAD001 in Advanced Neuroendocrine Tumours, Third Trial (RADIANT-3) Study Group: Everolimus for advanced pancreatic neuroendocrine tumours. N Engl J Med 2011;364:514-523.

4 Chan JA, Stuart K, Earle CC, Clark JW, Bhargava P, Miksad R, Blaszkowsky L, Enzinger PC, Meyerhardt JA, Zheng H, Fuchs CS, Kulke MH: Prospective study of bevacizumab plus temozolomide in patients with advanced neuroendocrine tumours. J Clin Oncol 2012; 30:2963-2968.
Neuroendocrinology 2017;105:394-402 DOI: 10.1159/000456035
401 
5 Yao JC, Phan AT, Chang DZ, Wolff RA, Hess K, Gupta S, Jacobs C, Mares JE, Landgraf AN, Rashid A, Meric-Bernstam F: Efficacy of RAD001 (everolimus) and octreotide LAR in advanced low- to intermediate-grade neuroendocrine tumours: results of a phase II study. J Clin Oncol 2008;26:4311-4318.

6 Raymond E, Dahan L, Raoul JL, Bang YJ, Borbath I, Lombard-Bohas C, Valle J, Metrakos $\mathrm{P}$, Smith D, Vinik A, Chen JS, Hörsch D, Hammel P, Wiedenmann B, Van Cutsem E, Patyna S, Lu DR, Blanckmeister C, Chao R, Ruszniewski P: Sunitinib malate for the treatment of pancreatic neuroendocrine tumours. N Engl J Med 2011;364:501-513.

7 Houk BE, Bello CL, Poland B, Rosen LS, Demetri GD, Motzer RJ: Relationship between exposure to sunitinib and efficacy and tolerability endpoints in patients with cancer: results of a pharmacokinetic/pharmacodynamic meta-analysis. Cancer Chemother Pharmacol 2010;66:357-371.

8 Kulke MH, Lenz HJ, Meropol NJ, Posey J, Ryan DP, Picus J, Bergsland E, Stuart K, Tye L, Huang X, Li JZ, Baum CM, Fuchs CS: Activity of sunitinib in patients with advanced neuroendocrine tumours. J Clin Oncol 2008; 26:3403-3410.

9 Pavel M, O’Toole D, Costa F, Capdevila J, Gross D, Kianmanesh R, Krenning E, Knigge U, Salazar R, Pape UF, Öberg K; all other Vienna Consensus Conference Participants: ENETS Consensus Guidelines Update for the Management of Distant Metastatic Disease of Intestinal, Pancreatic, Bronchial Neuroendocrine Neoplasms (NEN) and NEN of Unknown Primary Site. Neuroendocrinology 2016;103:172-815.

10 National Comprehensive Cancer Network (NCCN): Clinical Practice Guidelines in Oncology, version 1.2015. Washington, National Comprehensive Cancer Network, 2015.

11 Pavel ME, Hainsworth JD, Baudin E, Peeters M, Hörsch D, Winkler RE, Klimovsky J, Lebwohl D, Jehl V, Wolin EM, Oberg K, Van Cutsem E, Yao JC; RADIANT-2 Study Group: Everolimus plus octreotide long-acting repeatable for the treatment of advanced neuroendocrine tumours associated with carcinoid syndrome (RADIANT-2): a randomised, placebo-controlled, phase 3 study. Lancet 2011; 378:2005-2012.

12 National Comprehensive Cancer Network (NCCN): Clinical Practice Guidelines in Oncology: Kidney Cancer, version 1.2013. Washington, National Comprehensive Cancer Network, 2013

13 Escudier B, Eisen T, Porta C, Patard JJ, Khoo V, Algaba F, Mulders P, Kataja V; ESMO
Guidelines Working Group: Renal cell carcinoma: ESMO Clinical Practice Guidelines for diagnosis, treatment and follow-up. Ann Oncol 2012;23:65-71.

14 Motzer RJ, Barrios CH, Kim TM, Falcon S, Cosgriff T, Harker WG, Srimuninnimit V, Pittman K, Sabbatini R, Rha SY, Flaig TW, Page R, Bavbek S, Beck JT, Patel P, Cheung FY, Yadav S, Schiff EM, Wang X, Niolat J, Sellami D, Anak O, Knox JJ: Phase II randomized trial comparing sequential first-line everolimus and second-line sunitinib versus firstline sunitinib and second-line everolimus in patients with metastatic renal cell carcinoma. J Clin Oncol 2014;32:2765-2772.

15 Andersen PK, Gill RD: Cox's regression model for counting processes: a large sample study. Ann Stat 1982;10:1100-1120.

16 Neychev V, Steinberg SM, Cottle-Delisle C, Merkel R, Nilubol N, Yao J, Meltzer P, Pacak K, Marx S, Kebebew E: Mutation-targeted therapy with sunitinib or everolimus in patients with advanced low-grade or intermediate-grade neuroendocrine tumours of the gastrointestinal tract and pancreas with or without cytoreductive surgery: protocol for a phase II clinical trial. BMJ Open 2015;19:5.

17 Yao JC, Lombard-Bohas C, Baudin E, Kvols LK, Rougier P, Ruszniewski P, Hoosen S, St Peter J, Haas T, Lebwohl D, Van Cutsem E, Kulke MH, Hobday TJ, O'Dorisio TM, Shah MH, Cadiot G, Luppi G, Posey JA, Wiedenmann B: Daily oral everolimus activity in patients with metastatic pancreatic neuroendocrine tumours after failure of cytotoxic chemotherapy: a phase II trial. J Clin Oncol 2010; 28:69-76.

18 Caplin ME, Pavel M, Ruszniewski P: Lanreotide in metastatic enteropancreatic neuroendocrine tumours. N Engl J Med 2014;371: 1556-1557.

19 Sorbye H, Strosberg J, Baudin E, Klimstra DS, Yao JC: Gastroenteropancreatic high-grade neuroendocrine carcinoma. Cancer 2014; 120:2814-2823.

20 Fine RL, Gulati AP, Krantz BA, Moss RA, Schreibman S, Tsushima DA, Mowatt KB, DinNET RD, Mao Y, Stevens PD, Schrope B, Allendorf J, Lee JA, Sherman WH, Chabot JA: Capecitabine and temozolomide (CAPTEM) for metastatic, well-differentiated neuroendocrine cancers: The Pancreas Center at Columbia University experience. Cancer Chemother Pharmacol 2013;71:663-670.

21 Motzer RJ, Escudier B, Oudard S, Hutson TE, Porta C, Bracarda S, Grunwald V, Thompson JA, Figlin RA, Hollaender N, Kay A, Rayaud A; RECORD-1 Study Group: Phase 3 trial of everolimus for metastatic renal cell carcino- ma: final results and analysis of prognostic factors. Cancer 2010;116:4256-4265.

22 Rosa R, Damiano V, Nappi L, Formisano L, Massari F, Scarpa A, Martignoni G, Bianco R, Tortora G: Angiogenic and signalling proteins correlate with sensitivity to sequential treatment in renal cell cancer. Br J Cancer 2013;109:686-693.

23 Lane HA, Wood JM, McSheehy PM, Allegrini PR, Boulay A, Brueggen J, Littlewood-Evans A, Maira SM, Martiny-Baron G, Schnell CR, Sini P, O’Reilly T: mTOR inhibitor RAD001 (everolimus) has antiangiogenic/vascular properties distinct from a VEGFR tyrosine kinase inhibitor. Clin Cancer Res 2009; 15: 1612-1622.

24 Kulke M: A randomized open-label phase II study of everolimus alone or in combination with pasireotide LAR in advanced progressive pancreatic neuroendocrine tumours (pNET): COOPERATE-2 trial. 12th Annu Eur Neuroendocr Tumour Soc Conf Diagn Treat Neuroendocr Tumour Dis, Barcelona, March 2015.

25 Kulke M, Niedzwiecky D, Foster N, Fruth B, Kunz P, Kennecke H, Wolin E, Venook A: Randomized phase II study of everolimus (E) versus everolimus plus bevacizumab in patients with locally advanced or metastatic pancreatic neuroendocrine tumours, CALGB 80701 (Alliance) (abstract). J Clin Oncol 2015;33(suppl);4005.

26 Pusceddu S, de Braud F, Concas L, Bregant C, Leuzzi L, Formisano B, Buzzoni R: Rationale and protocol of the MetNET-1 trial, a prospective, single center, phase II study to evaluate the activity and safety of everolimus in combination with octreotide LAR and metformin in patients with advanced pancreatic neuroendocrine tumours. Tumouri 2014;100: 286-289.

27 Yao JC, Fazio N, Singh S, Buzzoni R, Carnaghi C, Wolin E, Tomasek J, Raderer M, Lahner H, Voi M, Pacaud LB, Rouyrre N, Sachs C, Valle JW, Delle Fave G, Van Cutsem E, Tesselaar M, Shimada Y, Oh DY, Strosberg J, Kulke MH, Pavel ME; RAD001 in Advanced Neuroendocrine Tumours, Fourth Trial (RADIANT-4) Study Group: Everolimus for the treatment of advanced, non-functional neuroendocrine tumours of the lung or gastrointestinal tract (RADIANT-4): a randomised, placebo-controlled, phase 3 study. Lancet 2016;387:968977.

28 Lee A, Chan DL, Wong MH, Li BT, Lumba S, Clarke S, Samra J, Pavlakis N: Systematic review on the role of targeted therapy in metastatic neuroendocrine tumor (NET). Neuroendocrinology, Epub ahead of print. 